\title{
Laser-based manipulation and readout for multi-ion traps in quantum computing (Conference Presentation)
}

E. Beckert, Christoph Waechter, Bernd Hoefer, Uwe Zeitner

E. Beckert, Christoph A. Waechter, Bernd Hoefer, Uwe Zeitner, "Laser-based manipulation and readout for multi-ion traps in quantum computing (Conference Presentation)," Proc. SPIE 11699, Quantum Computing, Communication, and Simulation, 116990I (15 March 2021); doi: $10.1117 / 12.2574608$

SPIE. Event: SPIE OPTO, 2021, Online Only 


\section{Laser-based manipulation and readout for multi-ion traps in quantum computing (Withdrawal Notice)}

E. Beckert, Christoph A. Waechter, Bernd Hoefer, Uwe Zeitner

Author Affiliations -

E. Beckert, ${ }^{1}$ Christoph A. Waechter, ${ }^{1}$ Bernd Hoefer, ${ }^{1}$ Uwe Zeitner ${ }^{1}$

${ }^{1}$ Fraunhofer Institute for Applied Optics and Precision Engineering - IOF (Germany)

Proceedings Volume 11699, Quantum Computing, Communication, and Simulation; 116990I

(2021) https://doi.org/10.1117/12.2574608

Event: SPIE OPTO, 2021, Online Only

Online Publication Date: 5 March 2021

Withdrawn from Publication: 12 March 2021

Publisher's Note: This paper, originally published 5 March 2021, was withdrawn per author request. 